\title{
Dynamics of large turbulent structures in a steady breaker
}

\author{
Javier Rodríguez-Rodríguez ${ }^{\mathrm{a}, \mathrm{b}, *}$, Carolina Marugán-Cruz $^{\mathrm{b}}$, Alberto Aliseda ${ }^{\mathrm{c}}$, Juan Carlos Lasheras ${ }^{\mathrm{a}}$ \\ ${ }^{a}$ Dept. of Mechanical and Aerospace Engineering, University of California, San Diego, 9500 Gilman Drive, La Jolla, CA 92093-0411, United States \\ ${ }^{\mathrm{b}}$ Fluid Mechanics Group, Universidad Carlos III de Madrid, Avd. Universidad 30, 28911 Leganés, Madrid, Spain \\ ${ }^{\mathrm{c}}$ Dept. of Mechanical Engineering, University of Washington, Stevens Way, Box 352600, Seattle, WA 98195, United States
}

\begin{abstract}
A B S T R A C T
The flow near the leading edge of a steady breaker has been studied experimentally using Bubble Image Velocimetry (BIV) with the aim of characterizing the dynamics of the large eddies responsible for air entrainment. It is well reported in the literature, and confirmed by our measurements of the instanta neous velocity field, that this flow shares some important features with the turbulent shear layer formed between two parallel semi infinite streams with different velocities. Namely, the formation of a periodic array of coherent vortices, the constant convective velocity of those vortices, the linear relation between their size and their downstream position and the self similar structure of both mean velocity profiles and Reynolds shear stresses. Nonetheless, important differences exists between the dynamics of the large eddies in a steady breaker and those in a free shear layer. Particularly, the convective velocity of these large structures is slower in a steady breaker and, consistent with this, their growth rates are larger. A physical interpretation of these differences is provided together with a discussion of their implications. To support our measurements and conclusions, we present a careful analysis of the accuracy of the BIV technique in turbulent flows with large bubbles.
\end{abstract}

\section{Introduction}

Turbulent spilling breakers or bores occur in a wide variety of flows relevant to both engineering and environmental applica tions, such as hydraulic jumps, flow in dam spillways, breaking waves in the ocean or the formation of bubbly wakes in ships. They are, however, far from being fully understood. The key difficulty is that the flow field close to the leading edge, or toe, is not easy to characterize using conventional measurement techniques in fluid mechanics nor is it easy to study using well established analytical or numerical tools. This is particularly true in strong breakers, that is in breakers where a properly defined Froude number is high en ough. This paper presents an experimental investigation conducted with the purpose of clarifying several aspects of these complex flows. In particular, a steady breaker configuration has been imple mented in our laboratory facility. In such configuration, the break er remains stationary with respect to the laboratory frame of reference, which facilitates the application of diagnostic tech niques and statistical tools. It should be kept in mind that the stea diness of the flow must be understood in a statistical sense, due to its turbulent nature.

* Corresponding author at: Fluid Mechanics Group, Universidad Carlos III de Madrid, Avd. Universidad 30, 28911 Leganés, Madrid, Spain.

E-mail address: fjrodriguez@ucsd.edu (J. Rodríguez-Rodríguez).
A significant amount of knowledge about the flow structure of steady breakers has been gathered in the past. It is commonly ac cepted that near the leading edge of the breaker, where the high speed stream impinges into a region of slower and deeper fluid, an unsteady two dimensional shear layer forms between the upper (nearly stagnant) and lower (fast) streams. This phenome non was first described by Peregrine and Svendsen [23], who also pointed out that even in flows where the bottom of the channel is close enough to affect the overall flow field, the initial develop ment of this mixing layer may be considered to be free from wall effects. This idea was further developed by Hoyt and Sellin [8], who investigated various similarities between the steady breaking in a hydraulic jump and a free mixing layer between two semi infinite streams. In their experimental investigation, they used high speed photographic techniques to show the existence of large coherent structures that grow linearly beneath the free surface. Unfortunately, since they did not measure velocity fields, very little quantitative information could be drawn from their results.

Velocity fields in steady breakers have been obtained experi mentally by several authors $[26,25,2,4,3,9,17,16]$. However, in highly aerated regions, difficulties to obtain precise measurements arise. In an attempt to avoid the difficulties of measuring in two phase flows, Rouse et al. [26] substituted one of the flat walls of a wind tunnel by a smooth wall reproducing the shape of the free surface of a hydraulic jump. This allowed the researchers to char acterize the turbulent velocity field with a hot wire anemometer. 
However, gravity effects, which are known to be key in any break er, are neglected in this experiment.

Accurate experimental characterization of the instantaneous velocity field in the shear layer found in a steady breaker was ob tained by Lin and Rockwell [10] from PIV measurements. This tech nique allowed them to clearly identify large coherent structures developing between the lower, high speed stream and the upper, slow moving body of fluid. Svendsen et al. [28] used laser Doppler anemometry to characterize the mean velocity field and the Rey nolds stresses in a steady breaker. They obtained mean velocity and shear stress profiles that clearly showed the existence of a shear layer. More recently Liu et al. [11] used Doppler ultrasound velocimetry to characterize the turbulent stresses in low Froude number hydraulic jumps, where the void fraction is small enough for this technique to operate properly. These authors found the maximum Reynolds stress at the center line of the mixing layer, where the turbulent intensities were greater than $20 \%$. Interest ingly, they also suggested that these shear stresses exhibit a self similar profile. Misra et al. [17] also investigated the structure of the shear layer in a weak hydraulic jump and measured a number of parameters of the shear layer that further support the analogy of this flow with homogeneous free shear layers.

The location of the mean free surface and its fluctuations in this type of flows, as well as the role that the turbulent structures have on it, has been studied under different conditions $[18,22,20]$. Experimental observations reveal that in strong turbulent breakers (such as those found in large hydraulic jumps) the collapse of the free surface is the leading mechanism of air entrainment. Misra et al. [17] tried to quantify the air entrainment along the whole length of a weak hydraulic jump, with a Froude number of 1.19 , using the fluctuations of the free surface. They observed that, in the region occupied by the hydraulic jump, the free surface exhib its large fluctuations that may be associated with the entrapment of air cavities. However, this result does not explain the precise physical mechanisms that lead to the collapse of the free surface and the resulting air entrainment.

Despite of all the available knowledge on turbulent breakers, there are several key aspects that are poorly understood. One of the most important in terms of both fundamental scientific under standing and technological application, is the mechanism by which air bubbles are entrained underneath the free surface. A significant fraction of the total air amount entrained by the breaker is known to occur at the leading edge $[3,21]$, where the flow separation oc curs, which is consistent with the flow structure proposed by Long uet Higgins and Turner [13] and Cointe and Tulin [4] among others. This mechanism is particularly important in weak breakers. However, as the breaker becomes stronger, the air entrainment oc curs all along the length of the breaker rather than being localized at the toe, although part of the entrainment still occurs at that loca tion. The large coherent structures that develop in the shear layer are energetic enough to produce overturning at the free surface, thus entrapping air cavities that are then broken up into smaller bubbles by the ambient turbulence $[12,19,20]$.

As the large coherent structures occurring in the shear layer are ultimately responsible for the collapse of the free surface and the subsequent entrapment of air cavities, it seems logical that any accurate model of air entrainment should be based on the knowl edge of the dynamics of these large vortices. This is precisely the aim of this paper, to experimentally characterize a number of fea tures describing the behavior of these structures, relating them to global parameters of the overall flow. Moreover, by studying the structure of the mean velocity field we describe some similarities and differences between the turbulent structures found in this flow and in free plane turbulent mixing layers. A deep water, weak Fro ude number (1.4 2.3) hydraulic jump set up was chosen as a con venient way to generate a steady breaker in the laboratory. Since our work focuses on the dynamics of the flow near the leading edge, where the bottom effects are negligible [23], most conclu sions of this investigation are of general nature, in that they can be applied to natural flows that share the same characteristics, such as spilling breakers or turbulent bores. This study could also be of interest to quantify certain aspects of man made flows such as that found at a ship stern.

The paper is organized as follows: Section 2 describes the experimental set up and the postprocessing techniques used to analyze the flow. The experimental results are presented in Section 3. Interpretation and discussion of the results is done in Section 4. Finally, Section 5 summarizes the main conclusions from this study.

\section{Experimental set-up}

The experiments were carried out in a recirculating water chan nel with a capacity of roughly five cubic meters. The test section was $2 \mathrm{~m}$ long and had a square cross section of $0.6 \mathrm{~m} \times 0.6 \mathrm{~m}$. The plenum was connected to the test section by a series of grids and honeycombs, followed by a contraction, to assure that fluctu ations originating at the pump are damped out before the flow reached the test section. The underlying turbulent intensity of the free stream measured from previous experiments [1] was very low, less than $0.5 \%$.

A plexiglass plate was cut to dimensions $0.6 \mathrm{~m} \times 0.0127 \mathrm{~m} \times$ $1 \mathrm{~m}$ and placed vertically across the test section of the water chan nel. An auxiliary horizontal plate was fixed to the upstream look ing side of the vertical plate at a shallow depth under the free surface (about $5 \mathrm{~cm}$ ), as is sketched in Fig. 1. In this way, the sur face oscillations and spurious bubbles entrained upstream of the region of interest were avoided. Once positioned inside the test section, the vertical flat plate extended through the entire width of the test section, from a distance of about $0.2 \mathrm{~m}$ (depending on the experimental session) from the bottom of the channel to well above the free surface. The end of the vertical plate that induced the hydraulic jump was machined to a sharp edge so that the free stream detaches cleanly from the gate without any possible boundary layer growth that would perturb the experiment and hinder its reproducibility. A sketch of the facility and the flow, are shown in Fig. 1.

The gate aperture together with the flow rate were used to vary the upstream flow velocity and water depth at the toe of the jump. For each experimental session, the free stream velocity was mea sured using a pitot tube. The tube was mounted on a rail system that allowed its displacement in the three directions with a posi tioning accuracy of $0.5 \mathrm{~mm}$. It must be pointed out that the velocity of the free stream was measured at different locations downstream of the gate under the region of interest to check that it remained constant, thus allowing us to discard any possible effect of the bot tom in the dynamics of the large coherent structures. More specif ically, we checked that the thickness of the boundary layer at the bottom of the test section was always smaller than $1 \mathrm{~cm}$ under the toe.

\subsection{Computation of the velocity fields and associated turbulent magnitudes}

Light scattered by the air bubbles entrained by the flow was captured by a Kodak ES 1.0 ( 1 Mpixel) digital camera at $180^{\circ} \mathrm{col}$ lection angle (first mode reflection). Illumination was provided by a strobe light positioned nearly coaxial with the optical axis of the camera. The camera was focused in a vertical plane aligned with the mean direction of the flow and located about $10 \mathrm{~cm}$ from the channel walls. Since the duration of the strobe light is extre 


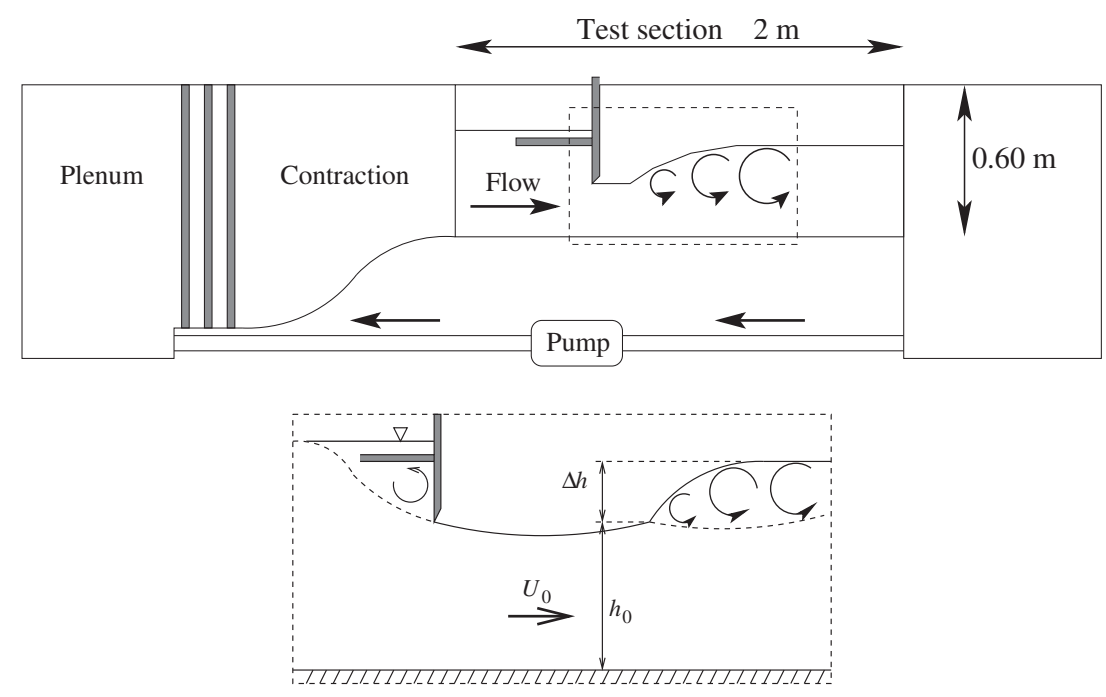

Fig. 1. Sketch and detail of the experimental facility.

mely short (about $2 \mu \mathrm{s}$ ), we expect no movement of the bubbles during that time. The lens used was a Nikkor Micro $60 \mathrm{~mm}$ with the aperture set at $f / 5.6$. The magnification ratio was approxi mately $1 / 16$ and, at this distance, the depth of field of these images was approximately $8 \mathrm{~cm}$, so bubbles between 6 and $14 \mathrm{~cm}$ from the channel wall were imaged and analysed.

In order to obtain information on the dynamics of the large coherent structures, correlation algorithms commonly used in Par ticle Image Velocimetry (PIV) were applied to the analysis of image pairs of the hydraulic jump acquired $\Delta t=1 \mathrm{~ms}$ apart. The synchro nization of the camera and the strobe light was performed with a Real Time Linux (RTAI) PC, which allows for accurate control of the timing. This technique, which has been referred to as Bubble Image Velocimetry (BIV) [27], is able to detect, although with some limitations, the rolling motion of the cloud of bubbles entrained by the large eddies, thus allowing the measurement of the velocity field wherever the bubble concentration is large enough. It must be pointed out that due to the nature of BIV, it is not suited to char acterize the fine scale velocity fluctuations of the flow. Therefore, the size of the interrogation window was chosen to be relatively large in order to average these small scale fluctuations. Thus, the velocity measurements correspond only to the velocity field asso ciated with the large scale eddies in the flow. All the experimental results described in this paper were obtained with an interrogation

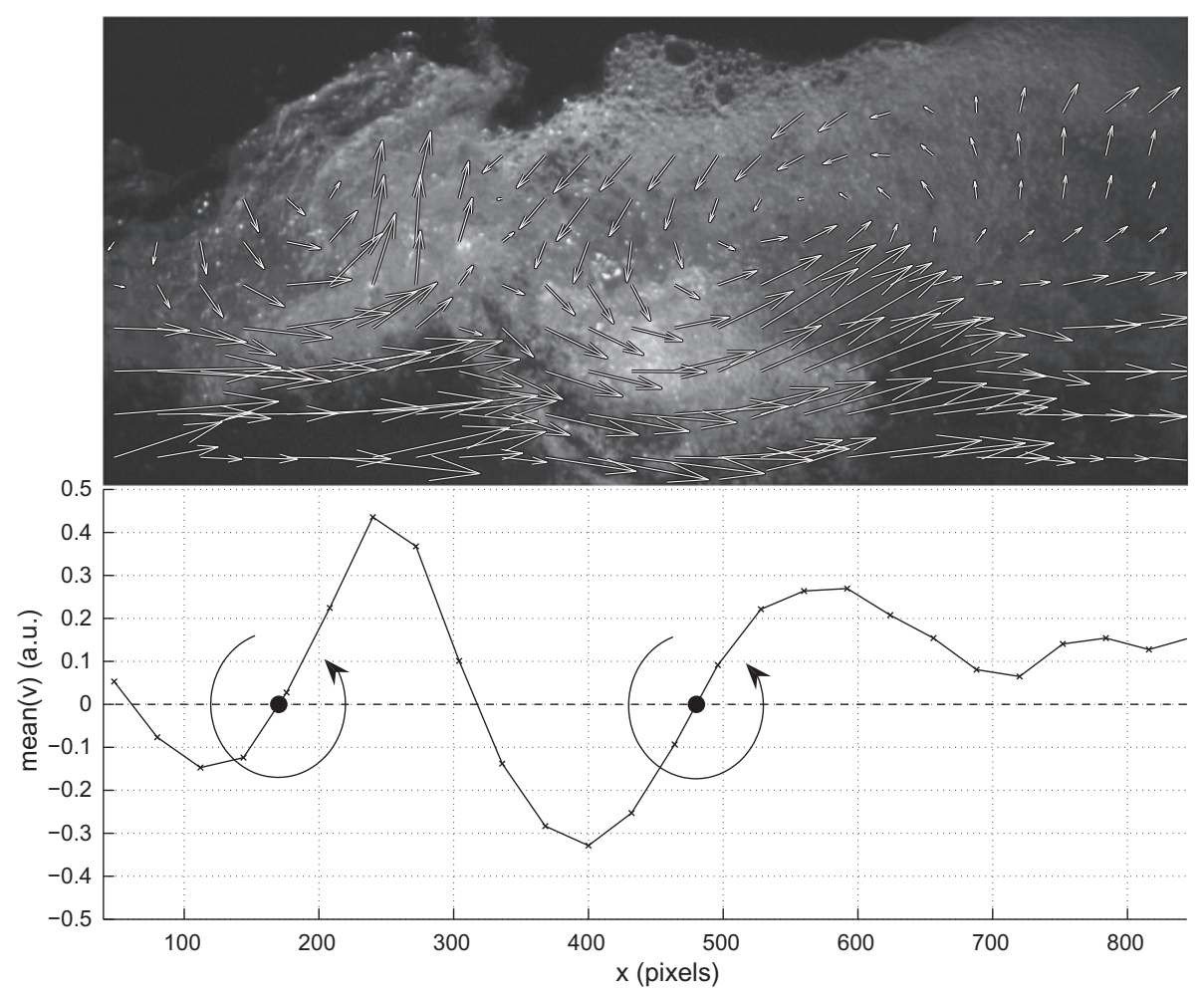

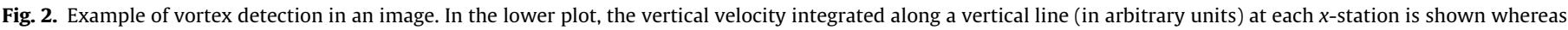
the circles represent the horizontal positions of the vortices detected by the algorithm. 
window of $64 \times 64$ (about $25 \mathrm{~mm} \times 25 \mathrm{~mm}$ ) pixels with a $50 \%$ overlap, which provided for about 10 velocity measurements across the diameter of a large eddy.

The same images used to compute the velocity field were also employed to measure the mean free surface. To do so, the instan taneous free surface was detected in each image by a thresholding method. The mean free surface was obtained by ensemble averag ing these curves. This technique allowed us, not only to compute the mean free surface, but also its standard deviation, which was about $\sigma_{h_{1}} \approx 1 \mathrm{~cm}$ for the water depth downstream the roller, $h_{1}$, in all the experimental conditions. This value has been used to compute the horizontal error bars shown in Fig. 7.

With the instantaneous velocity fields measured with the BIV technique, the mean velocity field and Reynolds shear stresses were computed by ensemble averaging. In order to prevent spuri ous velocity vectors from contaminating the mean turbulent fields, two filtering criteria were applied to the instantaneous measure ments: (i) the instantaneous free surface measured in each image was used to exclude from the averages those velocity vectors cor responding to interrogation windows above this surface. (ii) For the velocities measured at each interrogation window and filtered according to criterion (i), a mean value and standard deviation was computed. Then, velocity vectors differing from the mean value in more than twice the standard deviation were considered to be out liers, and thus were excluded from the statistics without being substituted by interpolated values.

\subsection{Detection technique of the large coherent vortices}

Although numerous techniques to determine the position of the center of a vortex are available in the literature, most of them in volve the calculation of the spatial derivatives of the velocity field. Unfortunately, the BIV technique used here does not provide the spatial resolution necessary to allow for the application of any of these techniques. However, in the case under consideration it is possible to take advantage of the slender nature of the flow to carry out the detection using an algorithm that involves integration of the velocity field rather than differentiation. Similarly to what hap pens in a turbulent free shear layer, the centers of the large eddies lay along a line nearly parallel to the unperturbed free stream, which coincides with the horizontal direction in the present flow. Thus, when evaluated along this line, the vertical velocity is ex pected to experience a change in sign precisely at the center of every vortex. Moreover, since the sense of rotation of the vortices in our set up (where the free stream flows from left to right) must always be counterclockwise, this change in sign must be from neg ative to positive values as one moves towards increasing values of $x$. To simplify the detection process and to make it more robust, we calculated the mean vertical velocity along constant $x$ lines. The change in sign of this integral quantity corresponds to the down stream position of the vortex center. An example of the detection performed with this criterion is shown in Fig. 2. The upper part of the figure is a snapshot of the flow with the velocity vectors overimposed and the lower graph shows the value of the average vertical velocity at constant downstream $x$ locations together with the detected positions of the vortices marked by circles. Finally, in order to avoid spurious zero crossings of the integrated vertical velocity due to small scale fluctuations to be taken as vortices, the detected positions were visually inspected and, when the detection was not successful, they were removed from the data.

\subsection{Estimation of the experimental error in the velocity measurements}

Using large bubbles as tracers to measure turbulent velocity fields limits the accuracy of the BIV technique with respect to con ventional PIV. This introduces the need to estimate the accuracy of
Table 1

Summary of experimental conditions. As sketched in Fig. $1, U_{0}$ and $h_{0}$ are the free stream velocity and water depth upstream of the jump whereas $\Delta h$ is the height jump. On the other hand, $u_{c}=U_{c} / U_{0}$ is the dimensionless convective velocity of the large vortices measured in the flow. Finally, $\mathrm{Fr}$ and $\mathrm{Fr}_{\Delta h}$ are Froude numbers defined using the upstream depth, $h_{0}$, and the jump height, $\Delta h$, respectively.

\begin{tabular}{llllllll}
\hline Exp. cond. & $U_{0}(\mathrm{~m} / \mathrm{s})$ & $h_{0}(\mathrm{~m})$ & $\Delta h(\mathrm{~m})$ & $\mathrm{Fr}$ & $\mathrm{Fr}_{\Delta h}$ & $\mathrm{Re}$ & $\begin{array}{l}u_{c}=U_{c} l \\
U_{0}\end{array}$ \\
\hline 1 & & & & & & & \\
2 & 1.60 & 0.126 & 0.110 & 1.44 & 1.54 & 195000 & 0.463 \\
3 & 1.95 & 0.119 & 0.142 & 1.81 & 1.66 & 225000 & 0.420 \\
4 & 2.30 & 0.126 & 0.177 & 2.09 & 1.74 & 281000 & 0.370 \\
5 & 2.21 & 0.110 & 0.145 & 2.13 & 1.85 & 236000 & 0.308 \\
& 2.48 & 0.120 & 0.175 & 2.29 & 1.89 & 288000 & 0.294 \\
\hline
\end{tabular}

the measurements reported based on this technique. Three sources of error are considered, namely buoyancy of the bubbles, response time of the bubbles to turbulent velocity fluctuations and non uni formity of the velocity field inside the interrogation window. No tice that these error sources are also present in conventional PIV, although in the latter their effect can usually be reduced by the right choice of tracer particles, seeding density and interrogation window size, something not possible here.

The effect of buoyancy in the velocity measurements is esti mated as follows: for air bubbles with sizes in the millimeter range rising in water, buoyancy induces a vertical velocity of order $V_{t} \sim(g D)^{1 / 2}$, where $D$ is the bubble diameter [15]. The bubble size distribution has been measured in a set of the same images used to perform BIV, yielding an average diameter $D_{a v e}=3.07 \mathrm{~mm}$ and a standard deviation $\sigma_{D}=1.12 \mathrm{~mm}$. Following Maxworthy et al. [15] (see Fig. 4 of that paper), terminal rise velocities for air bub bles with sizes $D_{m} \pm \sigma_{D}$ in purified water are in the range $V_{t} \sim 0.25 \pm 0.05 \mathrm{~m} / \mathrm{s}$. Comparing these figures with the values re ported in Table 1, buoyancy effects induce vertical velocities on the bubbles of the order of $1020 \%$ of the free stream velocity, $U_{0}$. In the upper part of the roller region, where horizontal veloci ties are of the order of $0.2 \mathrm{~m} / \mathrm{s}$ (see Fig. 5), the theoretical vertical velocity of the bubbles has a magnitude comparable to the mea sured horizontal velocity. Vertical bubble velocities of the same or der as the horizontal convective velocity are not, however, measured in the near surface region, leading us to hypothesize that the measured horizontal velocity is influenced by buoyancy to a much smaller degree through its non linear contribution to the drag. Moreover, the error estimation tends to overestimate its va lue as there are two factors that reduce the difference between the bubble vertical velocity and the liquid one. The first factor is that experiments reported in [15] correspond to air bubbles in perfectly clean water, but in our study tap water was used, so smaller termi nal velocities must be expected due to surfactant accumulation on the bubble surface. On the other hand, since the void fraction in the upper part of the roller region is large, one would expect significant two way coupling between the liquid and bubbles, and therefore the measured velocity is expected to represent the mean flow velocity with an error smaller than the values computed above.

A second cause of error in BIV is due to the finite response time of bubbles to fluctuations in the flow velocity. This effect limits the size of the smallest velocity scale that can be characterized using this technique. In order to estimate the response time of a bubble, we will apply order of magnitude estimation to the equation of motion for a spherical bubble in a non uniform flow (Eq. (17) in [14]), simplified for a density ratio $\rho_{b} / \rho_{f} \ll 1$ :

$\frac{1}{2} \rho V_{b} \frac{\mathrm{d} \mathbf{v}}{\mathrm{d} t} \quad \frac{1}{2} \rho C_{D} A_{b}\left(\begin{array}{ll}\mathbf{u} & \mathbf{v}\end{array}\right)|\mathbf{u} \quad \mathbf{v}|+\frac{3}{2} \rho V_{b} \frac{\mathrm{D} \mathbf{u}}{\mathrm{D} t}$,

where $\mathbf{u}$ and $\mathbf{v}$ are the liquid and bubble velocity respectively, $A_{b}$ the bubble cross sectional area, $V_{b}$ its volume and $C_{D}$ a drag coefficient that is of order unity for the range of bubble Reynolds numbers 
found here. To simplify the estimation of the slip between the bub bles and the turbulent fluctuations, buoyancy effects, lift force and Basset force have been neglected in Eq. (1).

Equating the order of magnitude of the left hand side term in Eq. (1) (the bubble inertia due to its virtual mass) with the first term in the right hand side (the drag) yields for the characteristic acceleration time of the bubble when exposed to a velocity fluctu ation of order, $u^{\prime}(\ell)$,

$t_{a c} \sim \frac{D}{u^{\prime}(\ell)}$

But the turnover time of a turbulent structure of size, $\ell$, is precisely $t_{o} \sim \ell / u^{\prime}(\ell)$, so the bubble will be able to respond faster than this turnover time, $t_{a c}<t_{o}$, when $D<\ell$. In other words, the bubble will be able to follow turbulent structures larger than its own size. Since the size of the interrogation window has to be much larger than the bubble size itself, this means that the finite response time of a bubble is not the limiting factor for the smallest size of turbulent structure that can be characterized with BIV.

The final source of error in our measurements of the velocity field is due to the size of the interrogation window in the PIV algo rithm. The velocity gradients in the flow that occur at scales smal ler than the interrogation window are missed in the statistical analysis of the images and therefore there is a random uncertainty in the velocity measurements that depends on the absolute size of the window (in pixels) and on the relative size compared to the turbulent structures measured. Using the vorticity thickness as an estimate of the maximum shear, we found $\partial u / \partial y \sim U_{0} /$ $0.5 \Delta h \sim 40 \mathrm{~s}^{1}$, and a corresponding displacement gradient $\Delta x /$ $\Delta y \sim 0.04$ pixels/pixel. Based on their analysis of PIV error ob tained from the velocity gradients at the scale of the interrogation window, Raffel et al. [24] found that for a $64 \times 64$ pixel window and this value of the gradient, the uncertainty in the measured dis placement (in an rms sense) is less than 0.4 pixels. Compared to an average displacement of 5 pixels, this represents a value of under $10 \%$, smaller or comparable to the other sources of error found in this application of PIV algorithms to bubbly flow images (BIV). Near the free surface the error is maximum, but in this region the measurements have multiple other sources of uncertainty and the values are used only in a qualitative sense.

In summary, the use of millimeter size bubbles to obtain veloc ity measurements in a turbulent flow represents an extension of the Particle Image Velocimetry ideas and algorithms that, while introducing significant sources of error, delivers a novel technique that is capable of characterizing complex multiphase flows that have defied quantitative study so far. This use represent a first step in understanding the process of air entrainment by the break up of a turbulent bore. The level of uncertainty in the measurements shows that it is not negligible but is limited to values that do not put into question the qualitative and most of the quantitative re sults and conclusions that we extract from this study. Without a doubt the higher level of uncertainty and the point in which the quantitative results are most subject to refinement is the estimate of the Reynolds stresses presented in Fig. 3 and, particularly, Fig. 9.

\section{Experimental results}

Five experimental conditions, summarized in Table 1, have been studied following the procedure described in the previous section. For each experiment, a Froude number can be defined as
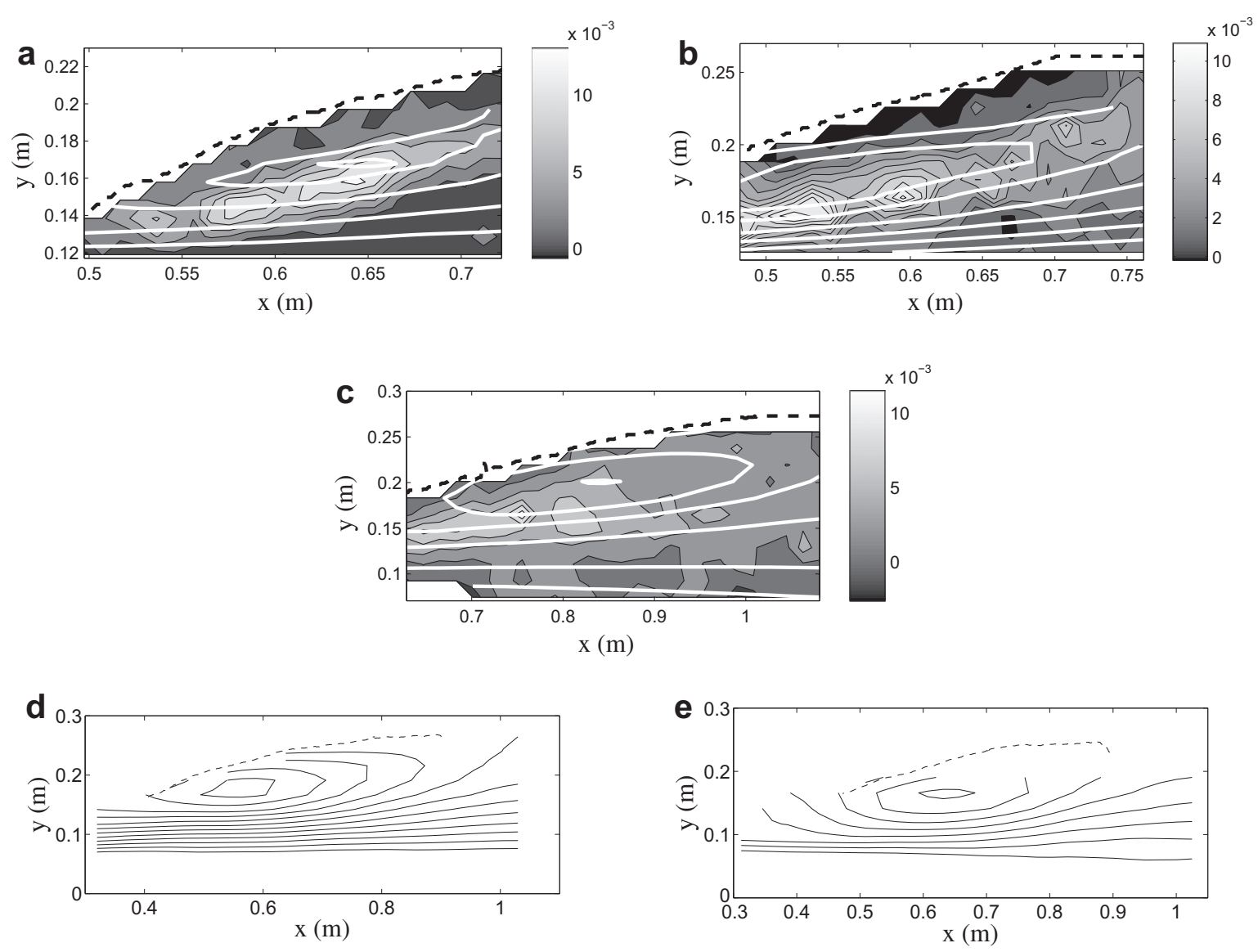

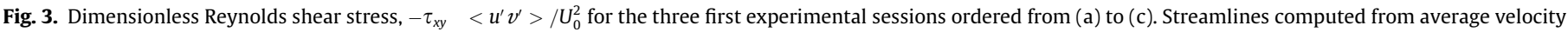
field have been superimposed (white lines) together with the average free surface (dashed line). For sessions 4 and 5 (panels e and d), only streamlines are plotted. 

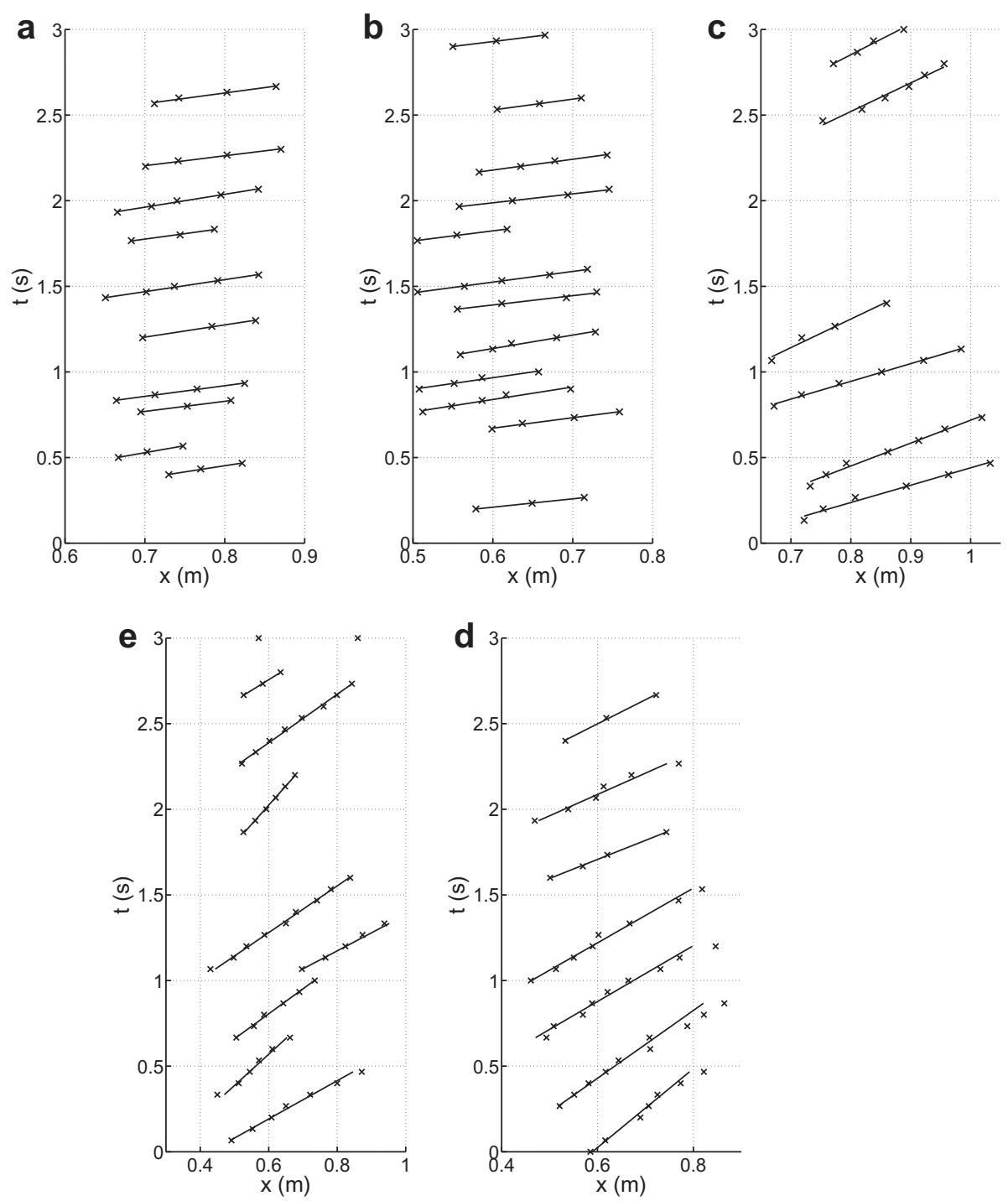

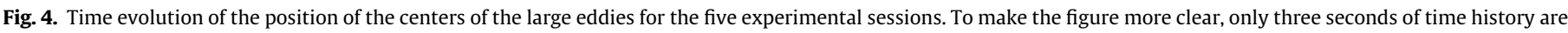
shown.

Fr $U_{0} / \sqrt{ } g h_{0}$, where $U_{0}$ is the free stream velocity, $h_{0}$ the up stream water depth and $g$ the gravitational acceleration. Addition ally, another Froude number based on the jump height, $\Delta h$, can be defined as $\operatorname{Fr}_{\Delta h} \quad U_{0} / \sqrt{ } g \Delta h$, and is shown in the same table. As will be discussed below, this second Froude number is more appropri ate to characterize several aspects of the mixing layer behavior and furthermore, since it is independent of the depth, $h_{0}$, results based on this Froude number can be applied more easily to steady breakers found in deep water flows.

The analysis of the instantaneous velocity fields obtained through BIV reveals the existence of large coherent vortices consis tent with the mixing layer structure described in the literature for both hydraulic jumps and spilling breakers $[23,8,10]$.

As pointed out by Peregrine and Svendsen [23], this mixing layer like behavior of the flow near the toe of the breaker is not incompatible with the classical picture of a steady roller structure. The streamlines computed from the mean velocity field are pre sented in Fig. 3. It can be seen that the roller, or recirculation re gion, emerges as a structure of the mean velocity field although it is not observed in any snapshot of the instantaneous velocity dis tribution. The Reynolds stresses in the breaking region, corre sponding to the area of the closed streamlines of the mean velocity, are also shown in Fig. 3 for the cases 13.

\subsection{Convective velocity of the large vortices}

An important feature of the large vortices that develop under neath the free surface is that they exhibit a nearly constant convec tive velocity, at least within the measurement region. In Fig. 4, the time evolution of the position of the centers of the large vortices is shown for the five experimental conditions investigated. From the $x \quad t$ trajectories of the centers, the mean convection velocities of the vortices, $U_{c}$, can be measured, yielding the values reported in Table 1 .

This constant convection velocity resembles the behavior of large coherent structures in a free turbulent mixing layer. However, it is important to point out an interesting difference: the measured con vective velocities do not correspond to the average between the fast and slow stream speeds. In our case, measurements show that the slow stream is nearly stagnant, in agreement with previous experi mental evidence [6], and therefore the expected convective velocity of the vortices $U_{c}$, if this shear layer was a homogeneous mixing layer, would be half of that of the free stream, $U_{0} / 2$. Interestingly, the vortices move always with velocities slower than $U_{0} / 2$ as can be seen in Table 1. Only in case 1, corresponding to the lowest Fro ude number investigated, $U_{c}$ approaches this value. The implications of this are discussed in the Section 4. 


\subsection{Growth rate of the mixing layer}

Another important feature of the dynamics of the vortices is their growth rate as they are advected downstream. Hoyt and Sellin [8] reported that, in their hydraulic jump experiment, large vortices grew linearly with the downstream distance until eventu ally they achieved a maximum size, resulting in a vortex structure analogous to that found in stratified mixing layers, where stratifi cation sets an upper limit to the growth of the overturning regions. They measured the growth rate of the vortices, and thus of the mixing layer, by drawing straight lines tangent to the vortices in still pictures of the flow. This method is too sensitive to the illumi nation conditions and, in some cases, fails to properly identify the lower limit of the mixing layer. This is specially true if air entrain ment is weak, when few bubbles are present. Therefore, in order to use a consistent method that could be applied to all the experi mental cases studied in this paper, we use a mixing layer thickness based on the average velocity field.

To illustrate the definition of mixing layer thickness that will be employed in this paper, a series of dimensionless horizontal veloc ity profiles have been plotted in Fig. 5. Two lines, $y_{0.1}(x)$ and $y_{0.35}(x)$, have been used to calculate the characteristic length scale: $\delta^{*}(x)=y_{0.1}(x) \quad y_{0.35}(x)$. These lines correspond to the vertical points of constant horizontal velocity: $y_{0.1}$, where $u\left(x, y_{0.1}\right) /$ $U_{0}=0.1$ and $y_{0.35}(x)$, where $u\left(x, y_{0.35}\right) / U_{0}=0.35$. These two values have been chosen to be as far apart as possible without getting too close to the free surface or to the bottom of the channel where too few bubbles are present in the experiment at the lowest Froude number. Velocities have been rescaled with the free stream value for each experimental condition, $U_{0}$, whereas the vertical coordi nate has been made dimensionless with the distance, $\delta^{*}(x)$. It is worth noting that the velocity profiles exhibit some degree of self similarity as one may expect in a mixing layer; although, a remarkable difference can be observed: in the region correspond ing to the roller, i.e. for $u / U_{0}$ close to zero, the velocity profiles are no longer self similar. We will come back to this point in the next section.

In order to compare our measurements of mixing layer thick ness with those reported previously in the literature, a vorticity thickness was calculated according to the following expression [5]:

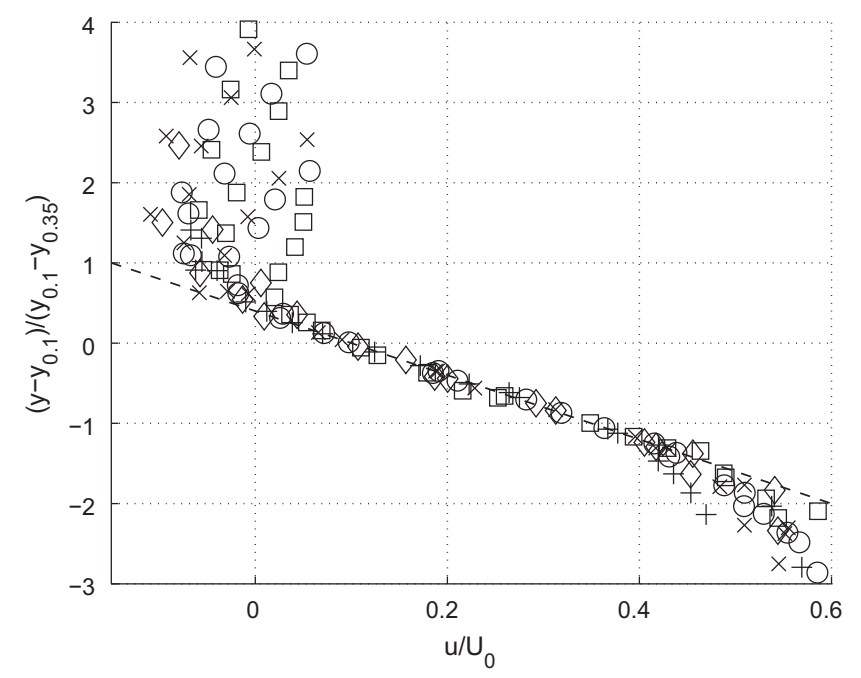

Fig. 5. Dimensionless velocity profiles within the region of linear growth for the five experimental cases. For the sake of clarity only three profiles from each case have been plotted, one close to the leading edge, one at the end of the recirculating bubble or roller and one midway through the region of interest. Symbols indicate experimental condition: $1(\times), 2(\square), 3(\circ), 4(+)$ and $5(\diamond)$.

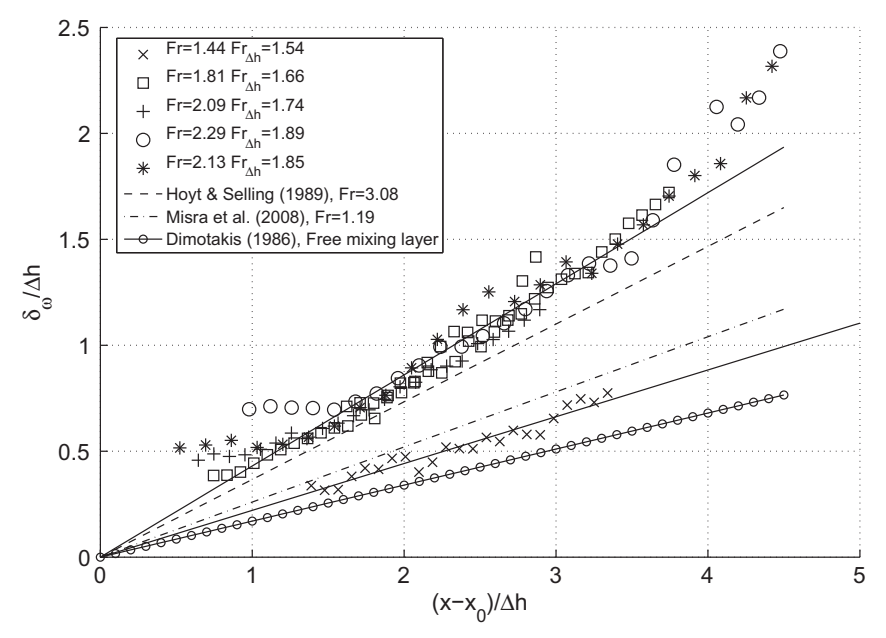

Fig. 6. Vorticity thickness measured for the five experimental sessions in Table 1. Dashed line corresponds to measurements by [8], dash-dot to [17] and dash-circle to the empirical correlation developed by [5].

$\delta_{\omega} \frac{U_{0}}{|\partial u / \partial y|_{\max }}$

In this definition, a velocity difference across the mixing layer equal to the free stream value was used. This is consistent with the fact that the velocity of the upper stream is very low, and its absolute value never exceeds $0.1 U_{0}$, as can be observed in Fig. 5 . We conclude, from the data shown in Fig. 5, that a good approximation to the maximum slope of the velocity profiles is $\left(0.1 U_{0} 0.35 U_{0}\right) / \delta^{*}$, corresponding to the dashed line. Therefore the vorticity thickness, $\delta_{\omega}$, can be related to the one actually measured, $\delta^{*}$, by $\delta_{\omega} \approx 4 \delta^{*}$. This vorticity thickness, non dimensionalized with the height of the jump, $\Delta h$, is plotted as a function of the dimensionless downstream distance for all the exper imental sessions in Fig. 6. Remarkably, except for the results of case 1, all the experimental conditions collapse into a single curve with a slope of approximately, $\delta_{\omega} /\left(\begin{array}{ll}x & x_{0}\end{array}\right) \approx 0.44$. Case 1 , corresponding to the lowest Froude number, $\mathrm{Fr}=1.44$, differs from all the other data in that its slope, $\delta_{\omega} /\left(\begin{array}{ll}x & x_{0}\end{array}\right) \approx 0.22$, is much closer to the value 0.17 proposed by [5] for a free shear layer forming between two semi infinite streams of equal densities, one moving with a speed, $U_{0}$, and the other one at rest.

\section{Discussion}

As shown in the previous section, the flow in the proximity of the toe of a steady breaker shares some features with a free shear layer, namely (1) the existence of an array of large coherent vortices that are convected downstream at constant speeds, (2) the linear growth of these large vortices (and therefore of the mixing layer), and (3) some degree of self similarity in the velocity profiles. Nonetheless, when quantitative comparisons are made between our measurements and those reported in the literature for homogeneous turbulent two dimensional mixing layers, some interesting differences arise.

\subsection{Convective velocity of the large eddies}

The first difference concerns the convective velocity of the large coherent structures. In the mixing layer developing in a steady breaker, eddies are slower than those observed in a free shear layer separating two streams of equal densities one at rest and the other moving with velocity $U_{0}$. In this latter case, the dimen sionless convective velocity would be $u_{c}=0.5$. In Fig. 7 , it can be seen that the dimensionless convective velocity, $u_{c}$, decreases with 


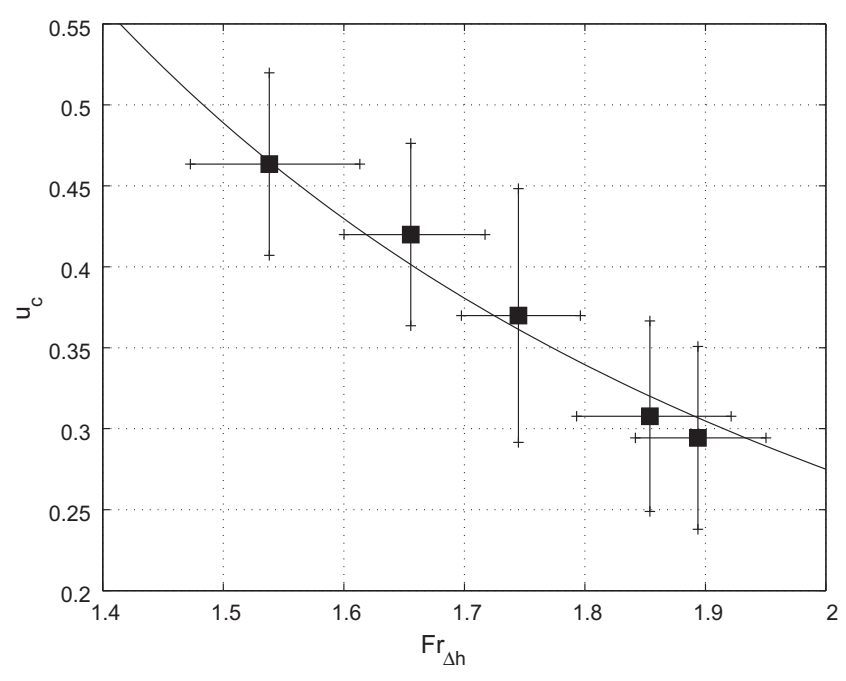

Fig. 7. Dependence of the dimensionless convective velocity of the large vortices, $u_{c}=U_{c} / U_{0}$, with the Froude number based on $\Delta h$. Experimental measurements are show as symbols, whereas the solid line corresponds to the fit $u_{c}^{\mathrm{fit}} 1.1 / \mathrm{Fr}_{\Delta h}^{2}$.

the jump height Froude number, with $u_{c}^{\mathrm{fit}} \quad 1.1 / \mathrm{Fr}_{\Delta h}^{2}$ being the best fit to the experimental measurements. This trend indicates that, for a constant speed of the fast stream, the thinner the mass of stag nant water resting on the shear layer, the slower the large vortices move. This conclusion is counter intuitive as one would expect the presence of a free surface, where shear stresses vanish, to speed up the vortices with respect to the case of the shear layer between two semi infinite streams. On the other hand, the slightly lower mean density of the spilling stream (due to the high void fraction found there) would also yield higher convective velocities of the vortices, since the average velocity of the two streams is weighted by their respective densities [5].

An interpretation of this result might be found following Dun can [6], who proposed that shear stresses acting along the dividing streamline that separates the spilling region from the fast stream, must balance the effect of gravity projected along the dividing streamline. According to another study [28], the dividing stream line that delimits the recirculation region falls approximately on the centerline of the shear layer, where maximum turbulent shear stresses occur, as can be seen in Fig. 3. Combining these ideas, one would conclude that an average shear stress acting along the divid ing streamline, $\tau$, should scale according to

$\tau \quad \rho\left\langle u^{\prime} v^{\prime}\right\rangle_{\max } \sim \rho g \Delta h \sin \alpha$,

where $\alpha$ is the angle between the dividing streamline and the hor izontal direction $\left(\alpha \approx 1018^{\circ}\right)$ [6]. On the other hand, provided that the dimensionless vortex convective velocity is inversely propor tional to the square of $\mathrm{Fr}_{\Delta h} \quad U_{0} / \sqrt{ } g \Delta h$, we can write

$u_{c} \sim \frac{g \Delta h}{U_{0}^{2}} \sim \frac{\left\langle u^{\prime} v^{\prime}\right\rangle_{\max }}{U_{0}^{2}} \frac{\tau}{\rho U_{0}^{2}}$,

indicating that the velocity of the large coherent structures is pro portional to the turbulent shear stresses along the mixing layer, that balance the hydrostatic pressure gradient existing across the jump. In order to verify the proposed relationship between turbu lent shear stresses at the centerline of the mixing layer and the thickness of the spilling water mass, turbulent Reynolds stresses computed for three experimental conditions are shown in Fig. 3 to gether with streamlines of the average velocity field. Consistently with measurements performed by Svendsen et al. [28] using La ser Doppler Anemometry and later by Misra et al. [17] using PIV, we also find that the maximum Reynolds shear stresses occur near

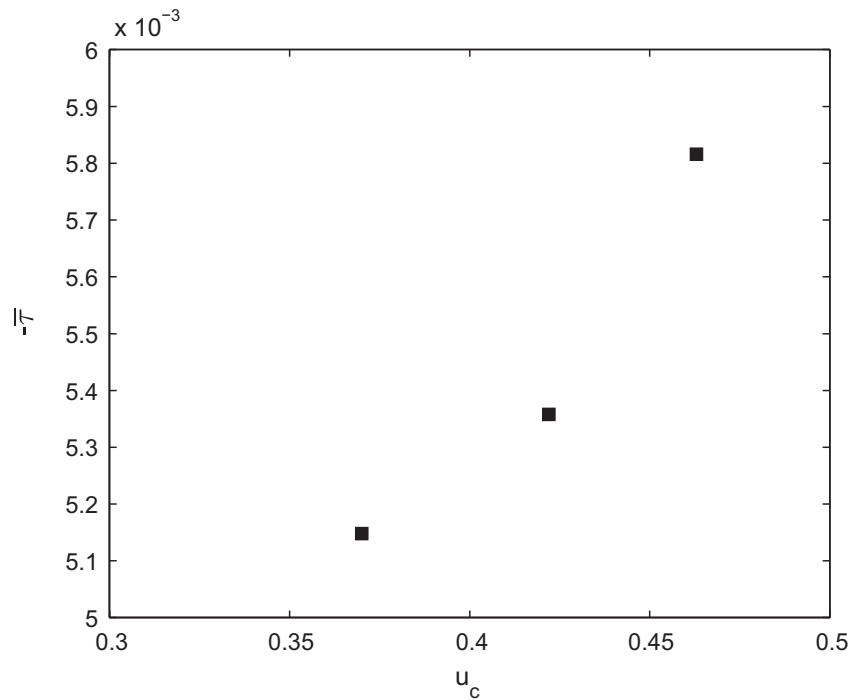

Fig. 8. Mean shear stress acting along the line of maximum stresses as a function non-dimensional convective velocity of the vortex for three experimental conditions.

the dividing streamline of the average velocity field. The average turbulent shear stress can therefore be calculated as a line integral along the curve where the maximum shear stresses lie:

$\bar{\tau} \quad \frac{1}{L} \int_{0}^{L} \frac{\left\langle u^{\prime} v^{\prime}\right\rangle_{\max }}{U_{0}^{2}} \mathrm{~d} \ell$,

where $L$ is the length of streamline used to evaluate the integral. The dimensionless average shear stress computed in this way (see Fig. 8) increases with the vortex convective velocity, $u_{c}$, thus sup porting the hypothesis that vortices move faster for higher values of the shear stress in the mixing layer. For lack of sufficient data, our measurements do not allow to verify with enough accuracy whether the relationship between $\tau$ and $u_{c}$ is in fact linear.

\subsection{Growth rate}

Pairing events between consecutive eddies were observed very rarely during the tracking of the large vortices. Therefore, entrain ment of irrotational fluid analogous to the one described by Herńan and Jiménez [7] and Dimotakis [5], among others, emerges as the primary mechanism responsible for the observed growth of the shear layer. At the same time, when the growth rates measured in this experiment are compared to the spread angle of a homoge neous free mixing layer forming between two streams with an equivalent velocity difference, higher values are obtained for all experimental conditions, as shown in Fig. 6. This is consistent with the fact that the velocity of the large eddies in the steady breaker is slower than in the free mixing layer. Only in the lowest Froude number experimental condition studied here (case $1, \mathrm{Fr}=1.44$ ), the measured growth rate, $\delta_{\omega} /\left(\begin{array}{ll}x & x_{0}\end{array}\right) \approx 0.22$, approaches the value of 0.17 proposed by Dimotakis [5] (circled line in Fig. 6).

To understand this, it is important to realize that, in the snap shots of the flow field in a weak spilling breaker shown by Lin and Rockwell [10], the large vortices of the mixing layer develop well below the free surface, so that very little interaction between this surface and the vortices may be expected. This also explains why the convective velocity of the vortices approaches $U_{0} / 2$ as the breaking becomes weaker. This picture is dramatically differ ent from strong breakers (see for example Fig. 2). In this latter case, vortices reach all the way up to the free surface, which therefore limits their growth. This geometrical limitation that the free 
surface imposes on the growth of the vortices is consistent with the collapse of the growth rate curves shown in Fig. 6 for $\mathrm{Fr}>1.8$. Remarkably, this transition occurs in a narrow range of parameters between $\mathrm{Fr} \approx 1.44$ and $\mathrm{Fr} \approx 1.8$. More measurements within this range are necessary to accurately describe this change of character in the behavior.

To better understand the generality of the observations made in this canonical flow and to validate our analysis, we compare our measurements with those published in the literature for similar flows. In Fig. 6, alongside our experimental results, we plot straight lines with the slopes measured by Hoyt and Sellin [8] and Misra et al. [17]. Hoyt and Sellin [8] reported, for a strong hydraulic jump with a Froude number of about 9, that the value of the slope is quite similar to the one we observe for all experimental conditions except case 1 . On the other hand, Misra et al. [17] performed anal ogous measurements for a weak hydraulic jump $(\mathrm{Fr} \approx 1.19)$. The value they reported is much closer to our result in case 1 , our low est Froude number. Although both papers use slightly different definitions of the shear layer thickness, comparison with their measurements seems to support our hypothesis that a rapid change of character occurs for these type of flows between $\mathrm{Fr} \approx 1.44$ and $\mathrm{Fr} \approx 1.8$, with an associated increase in the mixing layer growth rate. In order to compare the growth rates from the different experiments, the mean velocity field above the mixing layer has been assumed to be zero, which introduces a small error estimated to be approximately $10 \%$.

\subsection{Self similarity}

To conclude this section we present some observations regard ing the self similar structure of the velocity profile in these station ary breakers. As shown in Fig. 5, horizontal velocity profiles exhibit some degree of self similarity, as occurs in free mixing layers. However, above the dividing streamline, corresponding to values of $u / U_{0}$ close to zero, velocity profiles no longer follow this self similar curve. Thus, the self similar behavior is observed in the unencumbered region of the breaker, where growth is not con strained, but not in the region that is under the influence of the free surface. Inside this region, and for each section, $x$, the velocity pro files depart from the self similar curve at the point where the hor izontal velocity reaches its minimum value, $u_{\min }(x) / U_{0}$. This minimum velocity (or, alternatively, maximum spilling or recircu lation velocity) has been seen to decrease (increase) linearly with the distance to the toe, with typical values of the order of $10 \%$ of the free stream velocity.

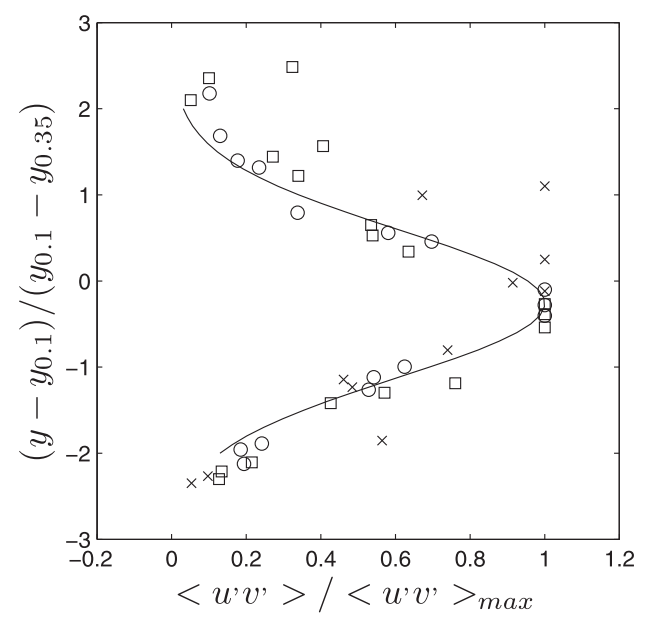

Fig. 9. Self-similar profiles, at different downstream sections, of the turbulent shear stresses corresponding to sessions $1(\times), 2(\square)$ and $3(\circ)$. The solid line is the result of fitting a gaussian curve to the experimental results.
We found that the Reynolds shear stresses exhibit self similar ity, as reported from experiments in a similar flow by Liu et al. [11]. The Reynolds stresses in our experiments have been calculated by subtracting the mean values of the horizontal and vertical velocity components to the instantaneous velocities and then calculating the ensemble average of the product of horizontal and vertical velocity fluctuations. In Fig. 9 we have plotted the Reynolds stres ses for experimental conditions 13 at the same downstream sec tions of the velocity profiles in Fig. 5. The resulting self similar shear stresses are well approximated by a gaussian distribution (solid line) as suggested by Townsend [29] for a free mixing layer. It is interesting to point out that the similarity variable employed here is not the one used by Liu et al. [11]. The difference is that the origin of our variable is a fixed reference of the mixing layer $\left(y=y_{0.1}\right)$ instead of the bottom of the channel, as Liu et al. [11] pro pose. This choice is not only preferable in order to apply our results to other steady breakers found in deep water flows, but also yields a better collapse in the dimensionless shear stress profiles, as can be seen comparing Fig. 9 in this work with Fig. 9 in Liu et al. [11].

\section{Conclusions}

The flow near the toe of a stationary breaker has been charac terized using Bubble Image Velocimetry (BIV) for a range of Froude numbers between 1.4 and 2.3. This technique has been used to quantify the dynamics of the large coherent vortices that dominate this type of flows as a first step to develop a physical model of air entrainment and bubble generation in steady breakers. Stationary breakers, turbulent bores and hydraulic jumps are known to devel op in a similar way, at least in the region near the leading edge, where most of the entrainment takes place.

Velocity measurements show that the flow in this region has many important similarities to the canonical turbulent mixing layer forming between two semi infinite parallel streams with dif ferent velocities, but two important differences emerge when quantitative comparisons are attempted. First, the large coherent vortices are convected downstream at a nearly constant speed; its value does not correspond, however, with the mean value be tween the maximum and the minimum horizontal velocities found in the flow, as is the case in a homogeneous free mixing layer. In stead, this velocity has been found to decrease approximately as the inverse of the square of a Froude number based on the jump height, suggesting that the presence of a free surface imposes a bal ance of stresses that is entirely different from the canonical mix ing layer. The equilibrium between gravity and shear stress necessary to establish the steadiness in the breaking process re quires a lower velocity of these coherent structures, and a corre sponding steeper slope of the dividing streamline. This steeper slope relates also to the second important difference: while the large coherent eddies, and therefore the shear layer flow under study, grow linearly with the downstream distance, the measured values of the growth rate are greater than those reported in the lit erature for a homogeneous free mixing layer. This is consistent with the low value of the vortex convective velocity found in the experiments. Only in the lowest Froude number conditions ana lyzed in this work, the growth rate was found to approach the va lue reported by Dimotakis [5] for free mixing layers. On the other hand, when the Froude number is greater than about 1.8 , the growth rate was found to approach a constant value, indicating that the presence of the free surface limits the size of the vortices. Regarding the growth mechanism of this shear flow, very few pair ing events have been observed, so that eddies grow mainly due to the engulfment of irrotational fluid. Within the region of linear growth, mean velocity profiles and shear Reynolds stresses exhibit a self similar behavior that only becomes apparent when appropri ate scales are introduced. 


\section{Acknowledgments}

The authors wish to thank Professor Emil J. Hopfinger for his valuable suggestions on the interpretation of the experimental data. This work was supported by the ONR through Grant N00014 0510121 and by the Spanish Ministry of Science (MIC INN) through Grant DPI2008 06369.

\section{References}

[1] A. Aliseda, J.C. Lasheras, Effect of buoyancy on the dynamics of a turbulent boundary layer laden with microbubbles, J. Fluid Mech. 559 (2006) 307-334.

[2] J.A. Battjes, T. Sakai, Velocity field in a steady breaker, J. Fluid Mech. 111 (1981) 421-437.

[3] H. Chanson, T. Brattberg, Experimental study of the air-water shear flow in a hydraulic jump, Int. J. Multiphase Flow 26 (4) (2000) 583-607.

[4] R. Cointe, M.P. Tulin, A theory of steady breakers, J. Fluid Mech. 276 (1994) 1 20

[5] P.E. Dimotakis, Two-dimensional shear-layer entrainment, AIAA J. 24 (11) (1986) 1791-1796.

[6] J.H. Duncan, An experimental investigation of breaking waves produced by a towed hydrofoil, Proc. R. Soc. Lond. A 377 (1981) 331-348.

[7] M.A. Hernán, J. Jiménez, Computer analysis of high-speed film of the plane turbulent mixing layer, J. Fluid Mech. 119 (1982) 323-345.

[8] J.W. Hoyt, R. Sellin, Hydraulic jump as a mixing layer, J. Hydr. Res. 115 (12) (1989) 1607-1614

[9] H.J. Leutherusser, J.J. Fan, Backward flow velocities of submerged hydraulics jumps, J. Hydr. Eng. 127 (6) (2001) 514-517.

[10] J.C. Lin, D. Rockwell, Evolution of a quasi-steady breaking wave, J. Fluid Mech. 302 (1995) 29-44.

[11] M. Liu, N. Rajaratnam, D.Z. Zhu, Turbulence structure of hydraulic jumps of low froude number, J. Hydr. Eng. 130 (2004) 511-520.

[12] D. Long, N. Rajaratnam, P.M. Steffler, P.R. Smy, Structure of flow in hydraulic jumps, J. Hydr. Res. 29 (2) (1990) 207-218.
[13] M.S. Longuet-Higgins, J.S. Turner, An 'entraining plume' model of a spilling breaker, J. Fluid Mech. 63 (1974) 1-20.

[14] J. Magnaudet, I. Eames, The motion of high Reynolds-number bubbles in inhomogeneous flows, Annu. Rev. Fluid Mech. 32 (2000) 659-708.

[15] T. Maxworthy, C. Gnann, M. Kurten, F. Durst, Experiments on the rise of air bubbles in clean viscous liquids, J. Fluid Mech. 321 (1996) 421-441.

[16] E. Mignot, R. Cienfuegos, Energy dissipation and turbulence production in weak hydraulic jumps, J. Hydr. Eng. 136 (2) (2009) 116-121.

[17] S.K. Misra, J.T. Kirby, M. Brocchini, F. Veron, M. Thomas, C. Kambhamettu, The mean and turbulent flow structure of a weak hydraulic jump, Phys. Fluids 20 (2008) 035106.

[18] M. Mossa, U. Tolve, Flow visualization in bubbly two-phase hydraulic jump, J. Fluids Eng. 120 (1998) 160-165.

[19] F. Murzyn, H. Chanson, Experimental assessment of scale effects affecting twophase flow properties in hydraulic jumps, Exp. Fluids 45 (2008) 513-521.

[20] F. Murzyn, H. Chanson, Surface fluctuations in hydraulic jumps, Exp. Thermal Fluid Sci. 33 (2009) 1055-1064.

[21] F. Murzyn, D. Mouaze, J.R. Chaplin, Optical fibre probe measurements of bubbly flow in hydraulic jumps, Int. J. Multiphase Flow 31 (2005) 141-154.

[22] F. Murzyn, D. Mouaze, J.R. Chaplin, Air-water interface dynamic and free surface features in hydraulic jumps, J. Hydr. Res. 45 (5) (2007) 679-685.

[23] D.H. Peregrine, I.A. Svendsen, Spilling breakers, bores and hydraulic jumps, in: Proc. 16th Int. Conf. Coastal Engng, Hamburg, vol. 1, 1978, pp. 540-550.

[24] M. Raffel, C. Willert, J. Kompenhans, Particle Image Velocimetry: A Practical Guide, Springer, 1998.

[25] F.J. Resch, J.J. Leutheusser, Reynolds stress measurements in hydraulics jumps, J. Hydr. Res. 10 (1972) 409-429.

[26] H. Rouse, M. Asce, T.T. Siao, S. Nagaratnam, Turbulence characteristics of the hydraulic jump, Trans., ASME 124 (1959) 926-966.

[27] Y. Ryu, K.A. Chang, H.J. Lim, Use of bubble image velocimetry for measurement of plunging wave impinging on structure and associated greenwater, Measure. Sci. Technol. 16 (2005) 1945-1953.

[28] I.A. Svendsen, J. Veeramony, J. Bakunin, J. Kirby, The flow in weak turbulent hydraulic jumps, J. Fluid Mech. 418 (2000) 25-57.

[29] A. Townsend, The structure of turbulent shear flows, second ed., Cambridge University Press, 1976. 